\title{
Expression of Concern to: Fetal Safety of Dydrogesterone Exposure in the First Trimester of Pregnancy
}

\author{
Gideon Koren ${ }^{1,2,3}$ (1) $\cdot$ Daniella Gilboa ${ }^{3} \cdot$ Rachel Katz $^{3}$
}

Published online: 8 January 2020

(c) Springer Nature Switzerland AG 2020

\section{Correction to: Clinical Drug Investigation https://doi.org/10.1007/s40261-019-00862-w}

The Editor-in-Chief of Clinical Drug Investigation has become aware that this article [1] contains a number of errors and inconsistencies. In addition, concerns have also been raised regarding the study design and statistical analysis. These concerns are being investigated and an update will be provided once a resolution has been reached. Until then the conclusions of the study should be interpreted with caution.

Gideon Koren and Daniella Gilboa did not agree with this notice; Rachel Katz was informed of the intention to place this notice but did not reply to correspondence regarding this.

\section{Reference}

1. Koren G, Gilboa D, Katz R. Fetal safety of dydrogesterone exposure in the first trimester of pregnancy. Clin Drug Investig. 2019. https://doi.org/10.1007/s40261-019-00862-w.
The original article can be found online at https://doi.org/10.1007/ s40261-019-00862-w.

Gideon Koren

gidiup_2000@yahoo.com

1 Adelson Faculty of Medicine, Ariel University, 40700 Ariel, Israel

2 Motherisk Israel Program, Zerifin, Israel

3 Kahn-Sagol-Maccabi Institute for Research and Innovation, Tel Aviv, Israel 